\title{
Strongyloidiasis: The Cause of Hypereosinophillia and Duodenal Ulcer in an Immunocompetent Individual
}

\author{
Hoque $\mathrm{MM}^{1}$, Sen $\mathrm{S}^{2}$, Uddin $\mathrm{MN}^{3}$
}

\begin{abstract}
Strongyloidiasis is a common parasitic disease in tropical and sub-tropical regions of the world. Infection with Strongyloides Stercoralis usually remains asymptomatic with peripheral eosinophilia and uncontrolled growth. Consequently, immunocompromised individuals are at a higher risk of complications of this disease. A case of an immunocompetent patient who had complaint of acute abdominal pain and was found to have duodenal ulceration. Laboratory examination revealed significantly elevated absolute eosinophil count at $17000 / \mathrm{cmm}$ (normal $0-500 / \mathrm{cmm}$ ). The stool R/E revealed rhabtidiform larvae suggestive of Strongyloides stercoralis nematode. Endoscopy of upper GIT showed ulcer in duodenum. The patient was treated with weekly dose of Tab Albendazole for two weeks and after that peripheral eosinophilia count became normal. This study found that the elevated eosinophil count played a central role in the pathogenesis.
\end{abstract}

\section{Introduction}

Strongyloidiasis is a parasitic disease caused by the Strongyloides stercoralis, an intestinal nematode of human. It is estimated that ten millions of people are infected worldwide, although no precise estimate is available ${ }^{1}$. Endemic region for Strongyloides infection include the Southeastern United States, Eastern Europe, Southeastern Asia, Bangladesh, Pakistan, sub-Saharan Africa, the West Indies and South America ${ }^{2}$. The clinical symptoms and signs reported in strongyloidiasis are frequently nonspecific ${ }^{3}$. In many patients it may be totally asymptomatic and so remains undiagnosed. But sometimes patient present with peripheral eosinophilia or may complain of myriad of symptoms including skin rash due to larval penetration, cough, wheezing, dyspnea, upper abdominal pain, nausea, vomiting or diarrhea. Strongyloides stercoralis induced gastrointestinal ulcer disease in immunocompromised patients has been well described in the literature ${ }^{4}$ but there are a very few reported cases of duodenal ulcer occurring in an immunocompetent individual due to Strongylides stercoralis infection ${ }^{5}$.

\section{Case Report}

A 22 years old soldier of Bangladesh Army was admitted to Combined Military Hospital (CMH), Bogra on 07/07/14 with constant abdominal pain for 1 week. The pain was located in umbilical region. He also developed black coloured stool one week after admission. The patient denied having any fever, chills, nausea, vomiting or altered bowel movements. There was no $\mathrm{H} / \mathrm{O}$ peptic ulcer disease, diabetes, renal failure, HIV infection; no H/O taking corticosteroids, immunosuppressive or anti-cancer therapy or NSAID ingestion. The patient provided $\mathrm{H} / \mathrm{O}$ swimming in a Pisci culture pond for 15 days, two and half month back prior to admission. On physical examination he was afebrile with normal blood pressure of 120/80 $\mathrm{mmHg}$ and a soft abdomen without any tenderness. Initial laboratory examination revealed an elevated white cell count $\left(24,000 / \mathrm{mm}^{3}\right.$; normal $4500-11000 /$ $\mathrm{mm}^{3}$ ) with a markedly elevated eosinophil count of 17000/ $\mathrm{mm}^{3}$ (normal 0-700 $\mathrm{mm}^{3}$ ). Upper GI Endoscopy showed ulcer in 1st part of duodenum. Stool for Occult blood test was positive on two occasions; stool R/E demonstrated the presence of larval form of $S$. stercoralis. The patient was treated with weekly dose of Tab. Albendazole for two weeks. After that peripheral eosinophil count reverted back to normal, abdominal pain diminished and stool regained normal brown colour. The patient was tested negative for HIV and ICT filariasis.

1. Lt Col Md. Monirul Hoque, MBBS, MCPS, DCP, FCPS, Classified Specialist in Pathology, CMH Bogra 2. Maj Shuvojit Sen, MBBS, FCPS, Department of Internal Medicine, CMH, Bogra 3. Brig Gen Md Nizam Uddin, MBBS, MCPS, DCP, FCPS, Associate Professor of Pathology, AFMC, Dhaka. 


\section{Discussion}

Human are generally infected by Strongyloides through the transcutaneous route. After dermal penetration, the filariform larvae, through undefined mechanisms, migrate to the small intestine. The most clinically relevant, migration is the classic pulmonary route, in which organisms enter the blood stream and are carried to the lungs, ascending the tracheobronchial tree to enter the gastrointestinal tract. An auto-infective cycle can occur at a low level throughout infection and allows subsequent generations to persist in the host indefinitely ${ }^{6}$. Upper gastrointestinal ulcer due to Strongyloides stercoralis infection is a rare entity in immunocompetent patients. Patients with a compromised immune system are predisposed to disseminated disease that involves multiple systems with subsequent possible septic shock. Patients with a history of human immunodeficiency virus (HIV) or human T-lymphotropic virus (HTLV-1) infection, malignancy, current chemotherapy, corticosteroid use, malnutrition, chronic pulmonary diseases, diabetes mellitus, or alcoholism are at a high risk of disseminated Strongyloides stercoralis ${ }^{7}$. Several mechanisms ${ }^{7,8}$ which involve immunosuppression of eosinophilic response and lymphocytic activation against the intestinal helminthes in combination with an altered intestinal motility createa nurturing environment for Strongyloides stercoralis maturing into an adult worm and invade the mucosal barriers of the gastrointestinal tract by filariform larvae. The immune response against the helminthic infestation is mainly controlled by the lymphocytes, namely, the T-helper cell type 2 lymphocytes with CD4 markers (TH2 cells), that secrete important cytokines, especially Interleukin-4 (IL-4), Interleukin-5 (IL-5), and Interleukin-10 (IL-10) in response to the helminthic exposure ${ }^{8}$. IL-4 induces an inflammatory process that promotes mast cell recruitment and intestinal goblet cell activation which alters gut physiology, ultimately dislodging the worm ${ }^{9}$. IL-5 plays a major role in the differentiation and maturation of the eosinophil, thus increasing eosinophil counts. The eosinophils carry toxic granules which contain major basic protein (MBP), eosinophil cationic protein (ECP), eosinophil derived neurotoxin (EDN), and eosinophil peroxidase (EPO) which are directly toxic to the larvae of Strongyloides stercoralis ${ }^{9,10}$. ECP and EDN possess ribonuclease activity that form pores into the membrane of target cells facilitating the entry of other toxic molecules into the cells with subsequent degeneration. Unfortunately, these toxic granules have cytotoxic effects on the gastrointestinal epithelium and may result in ulcer formation ${ }^{10}$.

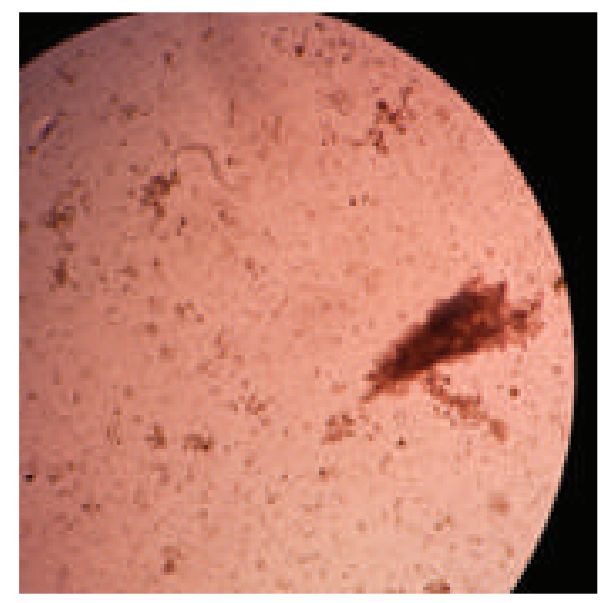

Fig-1: Stool Microscopy shows the larvae of Strongyloides stercoralis(40x).

High eosinophil counts in the serum should prompt the clinician to screen for parasitic disease. If there is still a strong suspicion, the absence of eosinophilia is not sensitive enough to rule out helminthic infections due to the fact that the eosinophils are mainly tissue dwelling cells ${ }^{9,10}$. Eosinophils are more numerous in tissue, a hundredfold more than in the peripheral blood. They are seen in body surfaces that have direct interaction with the environment like the respiratory tract, gastrointestinal tract (except esophagus) and lower genitourinary tract $^{9}$. In a study by Loutfy et $\mathrm{al}^{11}$, sixty-nine of seventy six patients positive for Strongyloides stercoralis as diagnosed by stool tests had peripheral eosinophilia. A case study reported by Shail et $\mathrm{al}^{5}$ showed elevated eosinophil count upto $11466 \mathrm{per} / \mathrm{cmm}$. In this study, the patient had total white blood cell (WBC) count of $24000 / \mathrm{cmm}$ and the calculated eosinophil count was $17000 / \mathrm{cmm}$, accounting for sixty three percent of all WBCs. Strongyloides stercoralis nematode infection induced a dramatic local inflammatory response in this patient as indicated by very high number of eosinophils. This extremely high number of eosinophils released large amount of toxic granules that produced ulceration in the upper gastrointestinal tract. 


\section{Conclusion}

This is the first case so far in Combined Military Hospital, Bogra of duodenal ulcer due to Strongyloides stercoralis infection in an immunocompetent patient with markedly elevated eosinophil cell counts and this study suggest that the eosinophil cells played a central role in the development of the ulcer.

\section{References}

1. Grove DI. Historical introduction. In: Grove DI (editor), Strongyloidiasis: A major roundworm infection of man. Philadelphia: Taylor \& Francis 1989:1-9.

2. Segarra-Newnham M. Manifestations, diagnosis, and treatment of Strongyloides stercoralis infection. Ann Pharmacother 2007 Dec; 41(12):1992-2001. Epub 2007 Oct 16.

3. Kim J, Joo H, Kim D et al. A case of gasric strongyloidiasis in a Korean patient. Korean J Parasitol 2003; 41(1):63-7.

4. Masud H and Sarkar MK. Hyperinfection with Strongyloides stercoralis in an Immunosuppressed Patient. Faridpur Med Coll J 2012; 2:98-101.
5. Sheth $S$ and Asslo F, Hallit R et al. Strongyloidiasis: The Cause of Multiple Gastrointestinal Ulcers in an Immunocompetent Individual. Hindawi Publishing Corporation, Vol-2014, Atricle ID 346256.

6. Neva FA. Intestinal nematodes of human beings. In FA. Neva editor. Basic clinical parasitology. Appleton \& Lange, Norwalk, Conn 1994; 123-8.

7. Keiser PB, Nutman TB. "Strongyloides stercoralis in the immunocompromised population," Clinical Microbiology Reviews 2004; 17:208-17.

8. Weller PE. Eosinophilia and eosinophil related disorders," in Middleton's Allergy and Immunology, chapter 49, Elsevier, 7th ed, 2008.

9. Zuo L, Rothenberg ME. "Gastrointestinal eosinophilia," Immunology and Allergy Clinics of North America 2007; 27(3):443-55.

10. Weller PE. The Immunology of Eosinophil's. The New England Journal of Medicine 1991; 324:1110-8.

11. Loutfy MR, Wilson M, Keystone JS et al. Serology and eosinophil count in the diagnosis and management of strongyloidiasis in a non-endemic area. American Journal of Tropical Medicine and Hygiene 2002; 66(6):749-52. 